Universida de Navarra

\section{TRANSCRIPTOMIC ANALYSIS OF EXTRACELLULAR VESICLES FOR BIOMARKER DISCOVERY IN PERIPHERAL ARTERY DISEASE (PAD)}

G. Saenz-Pipaon ${ }^{1,2}$ (gsaenzdepip@alumni.unav.es), D. Lara -Astiaso ${ }^{3}$, A. Maillo ${ }^{4}$, A. Vilas-Zornoza ${ }^{3,5}$, N. Planell4, J.A. Rodriguez $^{1,2,6}$, P. San Martín ${ }^{3}$, J.A. Paramo ${ }^{1,2,6,7}$, F. Prosper $2,3,4,7$, J. Orbe ${ }^{1,2,6}$, D. Gomez-Cabrero ${ }^{4}$, C. Roncal ${ }^{1,2,6}$.

(1) Laboratory of Atherothrombosis, Cima Universidad de Navarra, Spain. (2) IdiSNA, Navarra, Spain. (3) Oncohematology Program, Cima Universidad de Navarra, Spain. (4)

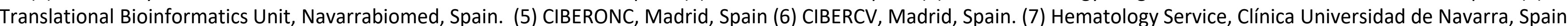

\title{
INTRODUCTION AND OBJECTIVES
}

\section{Peripheral artery disease}

- Major cause of chronic vascular pathologies in developed countries.

- High risk of developing cardiovascular (CV) events.

- Need of new molecular targets and/or prognostic tools.

\section{Lower limb}

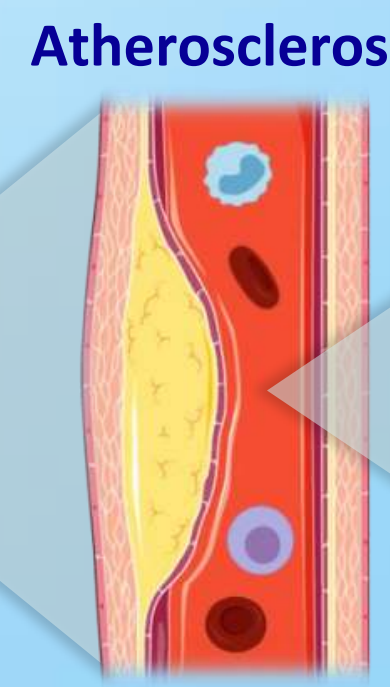

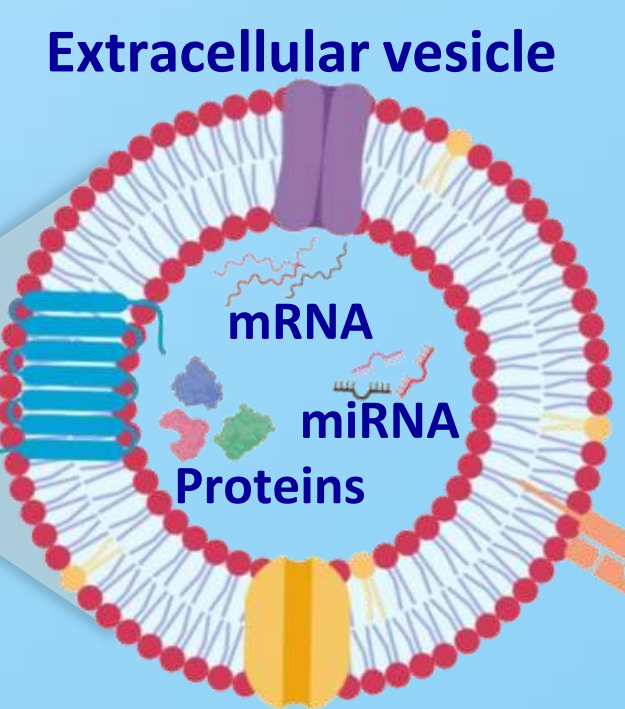

\section{Extracellular vesicles}

- Known as messengers for intercellular communication.

- Are augmented in subjects with CV risk factors.

- Their composition (liquid biopsy) could represent a valuable biomarker.

\section{METHODS}

\section{Isolation of EVs}

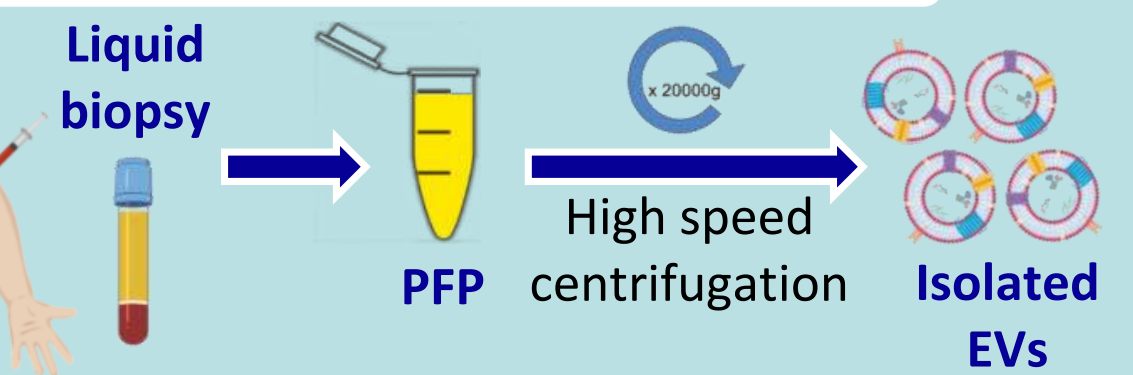

2 scRNA-seq library generation

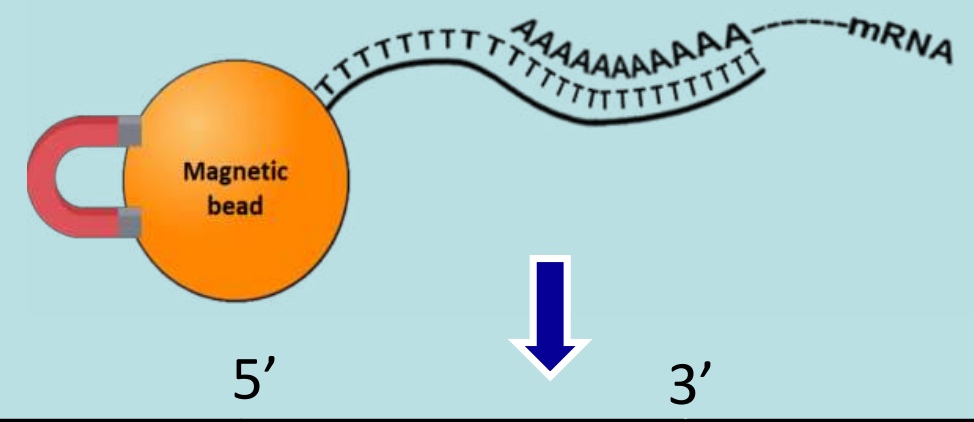

\begin{tabular}{|l|l|l|l|}
\hline Adapter & mRNA fragment & Barcode + UMI & Adapter \\
\hline
\end{tabular}

3 Bioinformatic analysis

Raw 3' scRNAseq data

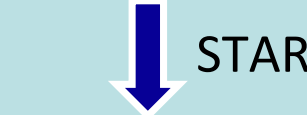

Mapped reads UMI-tools
quant $3 p$

Read counts
Low count genes filtering and normalization

\section{RESULTS}

1 Differentially expressed genes from the transcriptomic analysis of EVs

Group
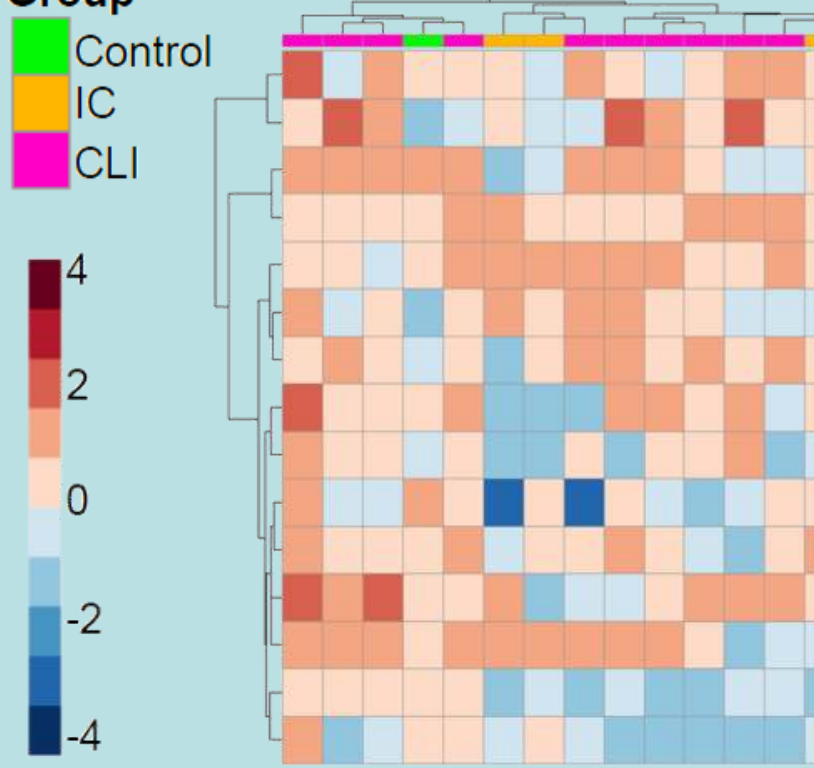

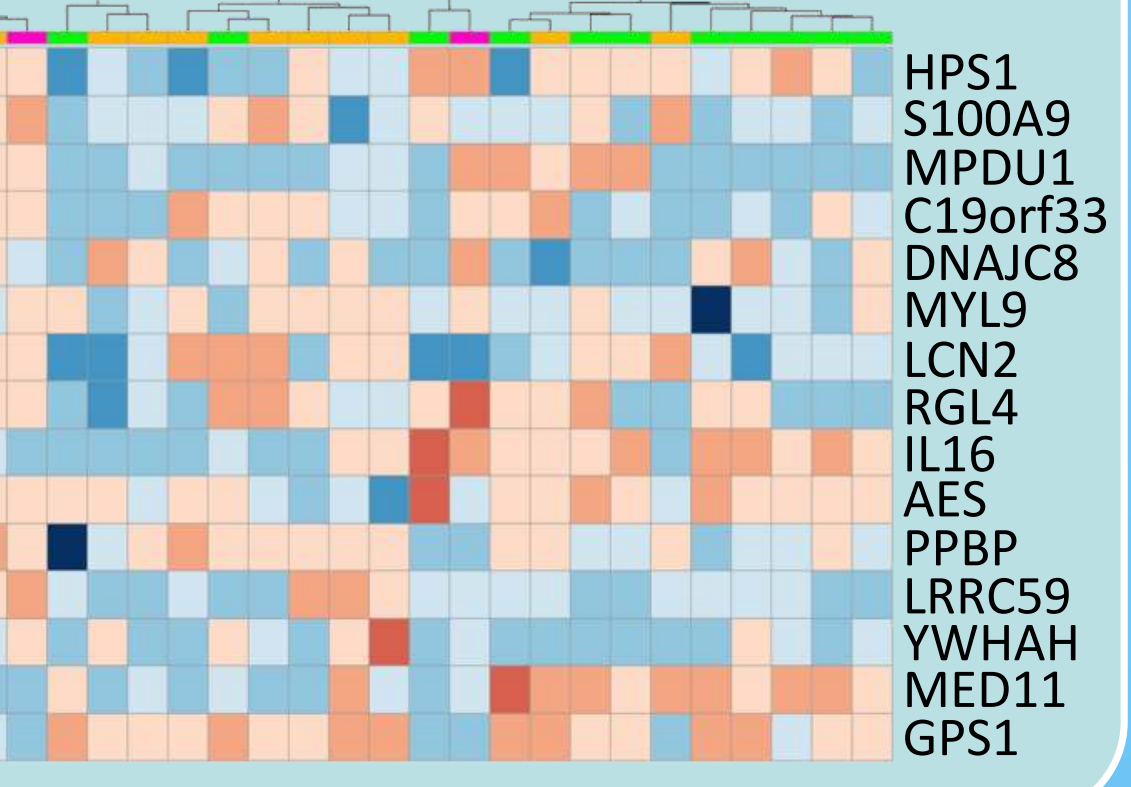

\section{CONCLUSIONS \& FUTURE PERSPECTIVES}

1. A protocol for studying the transcriptome of circulating EVs has been established.

2. 15 differentially expressed genes have been identified in EVs of PAD patients.

3. An association between calprotectin and amputation in PAD has been observed. Sorting of EVs by
cellular origin $\longrightarrow \begin{gathered}\text { Sequencing } \\ \text { (scRNA-seq) }\end{gathered} \longrightarrow \begin{gathered}\text { Identify and validate new } \\ \text { prognostic biomarkers }\end{gathered}$
2 Circulating calprotectin levels are correlated with EVs S100A9 expression and higher in PAD patients
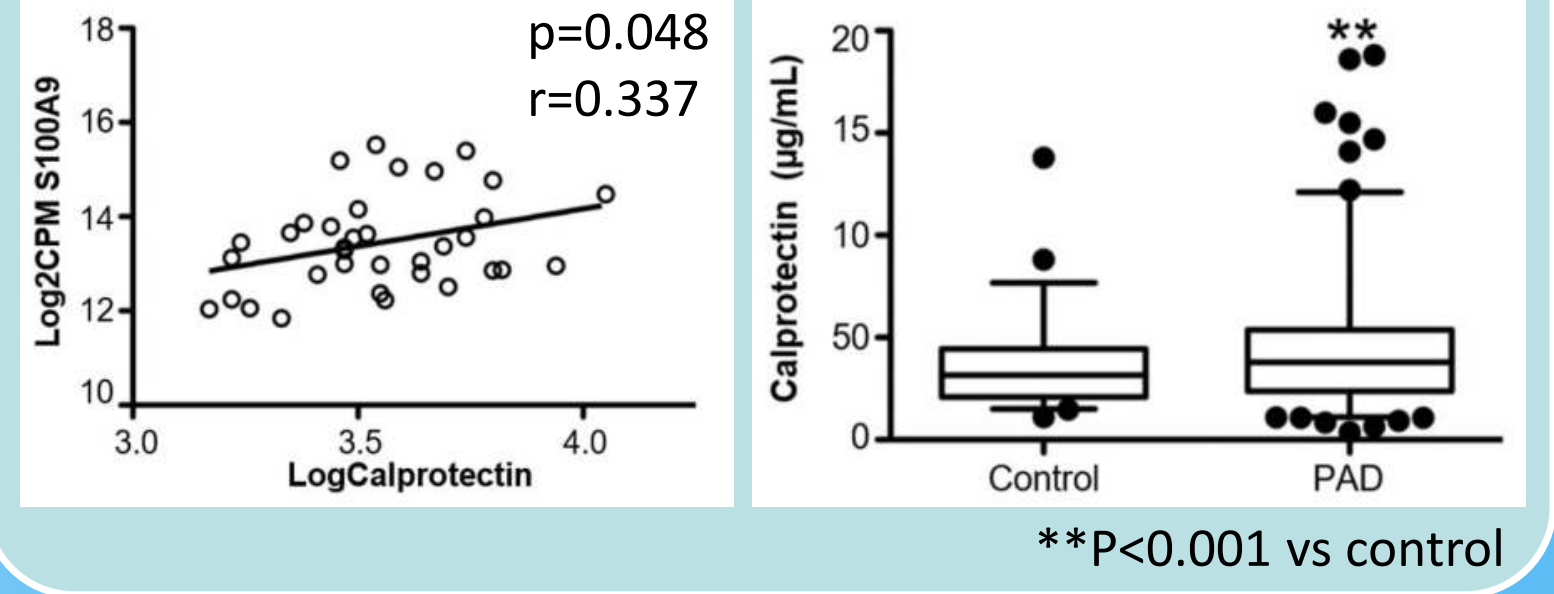

Calprotectin levels are associated to amputation in the follow-up

\begin{tabular}{lccc}
\hline & \multicolumn{3}{c}{ Calprotectin $(\mu \mathrm{g} / \mathrm{mL})$} \\
& SHR & 95\% Cl & P \\
\hline Amputation & & & \\
Unadjusted & 2.49 & $1.54-4.04$ & $<0.001$ \\
Model 1 & 2.56 & $1.56-4.19$ & $<0.001$ \\
Model 2 & 2.62 & $1.58-4.34$ & $<0.001$ \\
Model 3 & 2.57 & $1.58-4.17$ & $<0.001$ \\
\hline
\end{tabular}

Model 1: sex, age. Model 2: diabetes mellitus, dyslipidemia. Model 3: HT, eGFR.

\section{IdisNA}

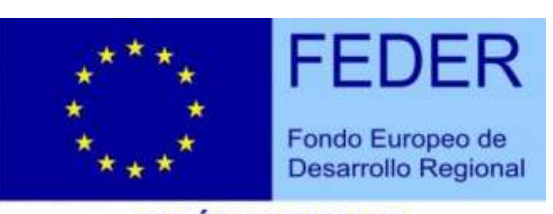
UNIÓN EUROPEA Una manera de hacer Europa"

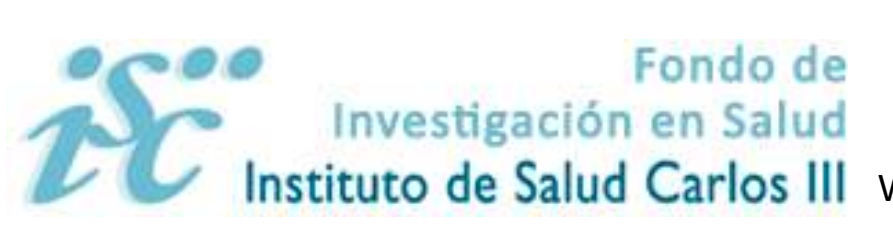

Instituto de Salud Carlos III
References

[1] Fowkes FG et al. Nat Rev Cardiol. 2017;14:156-170.

[2] Jansen et al. Circulation Research. 2017;120:1649-1657. 